\title{
Experimental measurement and Numerical Simulation of Particle Deposition on super-hydrophobic surface
}

\author{
Anjian Pan ${ }^{1}$, Lizhi Zhang ${ }^{1,2, *}$, and Hao Lu ${ }^{1, *}$ \\ ${ }^{1}$ School of Chemistry and Chemical Engineering, South China University of Technology, 510000 Guangzhou, People's Republic of \\ China \\ ${ }^{2}$ Key laboratory of Enhanced Heat Transfer and Energy Conservation of Education Ministry, 510000 Guangzhou, People's Repubilc of \\ China
}

\begin{abstract}
Airborne dust deposition on a large number of energy devices would cause serious efficiency and lifetime reduction, such as solar photovoltaic panels, heat exchanger surfaces and fan blades. Mechanical or manual cleaning using water is still the main method of mitigating dust deposition damage on these related energy equipment, which is commonly expensive and frequent. As a kind of self-cleaning material, super-hydrophobic coating may become a new effective way to mitigating the dust deposition issue. Super-hydrophobic coatings with low surface energy and unique micro-nano secondary structure can significantly reduce dust deposition rate. However, mechanism of dust deposition on super-hydrophobic surfaces remains unclear. Thus it is difficult to develop high performance self-cleaning coating. This paper aims to investigate dust deposition behaviors and mechanisms on super-hydrophobic surface by experimental measurement and numerical simulation. Lattice Boltzmann Method-Discrete Particle Method (LBM-DPM) will be developed to predict dust particle deposition process including settling, collision, adhesion and rebound behaviors. The mechanisms and interactions between coating surface energy, particle characteristics, particle incident velocity and particle adhesion or rebound behavior will be studied carefully. The findings and results may be useful to guide the development of high performance self-cleaning superhydrophobic coating.
\end{abstract}

\section{Introduction}

Airborne dust deposition on the surfaces of energy devices would cause serious problems in our daily life. For example, glass is widely used in solar system to protect the internal equipment. The spectral transmittance of the covering glass can significantly influence the PV output power efficiency [1]. However, dust deposition on the solar covering glass could reflect and absorb the sunlight, which would greatly reduce the transmittance of the glass and the efficiency of solar system [2]-[4]. For the semi-conductor manufacturing, a small amount of dust may destroy the integrated circuit. In some manufacturing industries such as food, medicine and petrochemical industry, dust deposition in the manufacturing process would affects the quality of products and cause potential danger. Therefore, dust removal technology need to be widely applied in many industries.

Mechanical or manual cleaning using water is still the main method of mitigating dust deposition damage on these related energy equipment, which is commonly expensive and frequent. Recently, super-hydrophobic coatings have become a new kind of self-cleaning materials to mitigate the dust deposition on the surfaces due to its low surface energy and micro-nano structures [5]. However, mechanism of dust deposition on superhydrophobic surfaces remains unclear. The influence of surface energy and micro-nano structure of superhydrophobic materials on particle deposition needs to be studied in detail. Thus this paper aims to investigate dust deposition behaviors and mechanisms on superhydrophobic surface by experimental measurement and numerical simulation. Lattice Boltzmann MethodDiscrete Particle Method (LBM-DPM) would be developed to predict dust particle deposition process including settling, collision, adhesion and rebound behaviors[6]-[7]. Moreover, high-speed micro camera will be used to record particle deposition process and validate the numerical results. The mechanisms and interactions between coating surface energy, particle characteristics, particle incident velocity and particle adhesion or rebound behavior will be studied carefully.

\section{Preparations of Materials}

Super-hydrophobic coatings were prepared by using organic silicone resin, ethanol and $\mathrm{SiO}_{2}$ nanoparticle in the experiment. The organic silicone resin and ethanol were mixed in a ratio of $1: 10$ and stirred at room

\footnotetext{
* Corresponding author: author@e-mail.org
} 
temperature. Then the $\mathrm{SiO}_{2}$ nanoparticles were added and stirred at room temperature for $4 \mathrm{~h}$. Organic silicone resin is used to reduce the surface energy while the $\mathrm{SiO}_{2}$ is used to create the micro-nano structure. After preparation of the super-hydrophobic coatings, the materials were evenly coated on a clean glass surface and dry for $10 \mathrm{~h}$. It can be seen from Fig. 1 that the contact angle on the surface is about $150^{\circ}$. Thus the glass surface is super-hydrophobic and it can be used for particle deposition experiment.

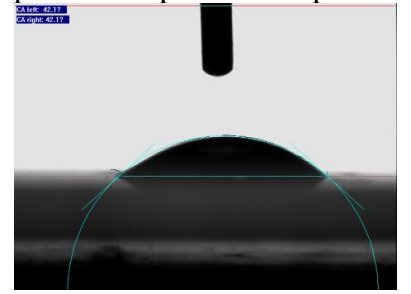

(a) Bare glass

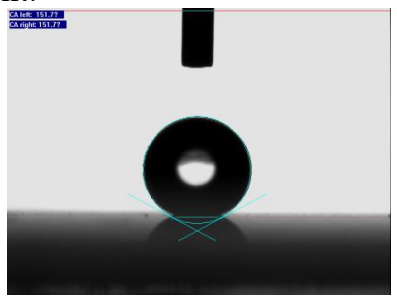

(b) Glass with coating
Fig. 1. The contact angles of different surface

\section{Computational Conditions and Numerical Model}

\subsection{Computational Conditions}

Deposition characteristic of single particle were investigated in the study. The computational condition can be seen in Fig. 2. Particle enters the horizontal pipe with a certain initial velocity and then the motion states would be changed due to the air drag force and gravity. Finally the particle would collide with the superhydrophobic surface. The velocity and height of the particle will be revealed in the simulation.

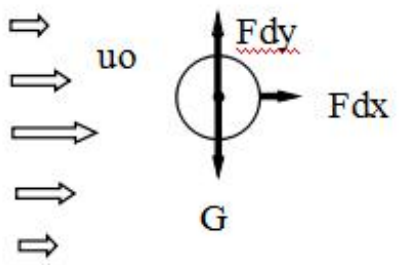

Fig. 2. The diagram of computational condition

In the simulation process, the left side is the inlet boundary condition while the right side is the fullydeveloped boundary condition, and the upper and lower walls are the no-slip boundary condition. The mesh size, fluid and particle parameters can be seen in Table1.

Table1. Parameters and Values Used In the Simulation

\begin{tabular}{cc}
\hline parameters & values \\
\hline Mesh size & $1.0 \mathrm{e}-4 \mathrm{~m}$ \\
Horizontal mesh number & 200 \\
Vertical mesh number & 100 \\
Diameters & $1.0 \mathrm{e}-3 \mathrm{~m} \sim 4.0 \mathrm{e}-3 \mathrm{~m}$ \\
Viscosity & $1.8 \mathrm{e}-5 \mathrm{~Pa} \cdot \mathrm{s}$ \\
Fluid density & $2.9 \mathrm{~kg} / \mathrm{m}^{3}$ \\
Particle density & $2500 \mathrm{~kg} / \mathrm{m}^{3}$ \\
\hline
\end{tabular}

\subsection{Numerical Model}

To obtain the particle deposition characteristic, the Lattice Boltzmann Method -Discrete Particle Method was selected in the simulation. The motion of the air flow is numerically evaluated using Lattice Boltzmann method. The LBM equations can be described as follows:

$$
f_{i}\left(x+e_{i} \delta_{t}, t+\delta_{t}\right)-f_{i}(x, t)=-\frac{1}{\tau}\left[f_{i}(x, t)-f_{i}^{e q}(x, t)\right]+F \cdot t
$$

Where $f_{i}(x, t)$ represents the fluid density distribution function and $\tau$ represents the relax time. The $F$ is the additional external force. The left side of the formula is stream step while the right side represents the collision step. The D2Q9 model and D3Q19 model are two common model for LBM simulation. Considering the computational efficiency and accuracy, the D2Q9 model was adopted in the study. The model can be seen as follows:

In the D2Q9 model, the equilibrium distribution function $f_{i}^{e q}(x, t)$ can be described as follows:

$$
f_{i}^{e q}(x, t)=\rho W_{i}\left[1+\frac{e_{i} u^{e q}}{C S^{2}}+\frac{\left(e_{i} u^{e q}\right)^{2}}{2 C S^{4}}-\frac{\left(u^{e q}\right)^{2}}{2 C S^{2}}\right]
$$

The weight coefficient $w_{i}$ are different for different direction. The $w_{0}, w_{1-4}$ and $w_{5-8}$ are 4/9, 1/9 and 1/36, respectively. The discrete velocity is given as follows:

$$
e_{i_{i}}=\left\{\begin{array}{l}
(0,0) \\
c \cdot\left(\cos \left[(i-1) \cdot \frac{\pi}{2}\right], \sin \left[(i-1) \cdot \frac{\pi}{2}\right]\right) \\
\left.\sqrt{2} \cdot c \cdot\left(\cos \left[(2 i-1) \cdot \frac{\pi}{4}\right], \sin (2 i-1) \cdot \frac{\pi}{4}\right]\right)
\end{array}\right.
$$

Where $c=\delta x / \delta$ tis the lattice speed. The fluid density and the velocity at each cell position can be calculated by

$$
\rho=\sum_{i} f_{i}, \quad \rho u=\sum_{i} e_{i} f_{i}
$$

The motion of the airborne particles can be obtained by solving the following equations according to Newton's second law

$$
\begin{aligned}
\frac{d \mathbf{x}_{p}}{d t} & =\mathbf{u}_{p} \\
m_{p} \frac{d \mathbf{u}_{p}}{d t} & =F_{d}+m g
\end{aligned}
$$

At low Reynolds number flow state, the drag force $F_{d}$ can be calculated:

$$
\begin{aligned}
F_{d} & =\frac{3 \pi d_{p} \mu}{C c}\left(u-u_{p}\right) \\
C c & =1+K n\left(1.257+0.4 e^{1.1 / K n}\right)
\end{aligned}
$$

\section{Results and Discussions}

\subsection{The distribution of the fluid flow}

Fig. 3 shows the flow distribution of the particle in a period of sedimentation - collision - rebound in horizontal pipe. A single particle enters the tube at a certain initial velocity from the inlet and then bounce off the bottom wall under the force of gravity and drag force. 
A certain structure formed in the tail of the particle can be seen in the figure due to the presence of particle.

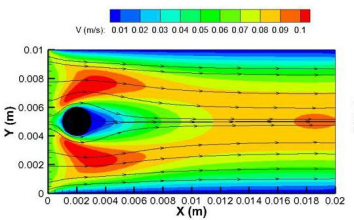

$\mathrm{t}=1.0 \mathrm{e}-3 \mathrm{~s}$

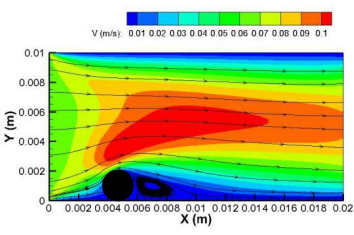

$\mathrm{t}=25.5802 \mathrm{e}-3 \mathrm{~s}$

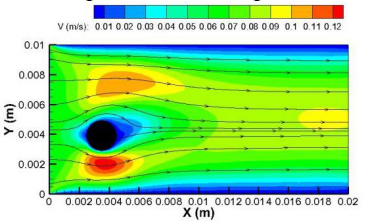

$t=16 e-3 s$

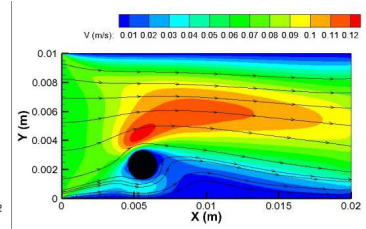

$t=36.75636 e-3 s$
Fig. 3. Flow distribution at different time

\subsection{The effects of parameters on particle deposition}

The diameter, elastic modulus of particles and surface energy of materials can greatly influence the particle deposition behaviors. Therefore, the influence of different factors on particle deposition behaviors was investigated in the study. The results can be seen in following parts.

\subsubsection{Effect of the surface energy on particle deposition}

The surface energy of materials can greatly influence the dust deposition on the super-hydrophobic surface. The influence of Van der Waals force between particle and super-hydrophobic surface was found and it can not be ignored during the particle deposition. The surface energy can affect the adhesion force between the particle and surface during particle deposition process. To specifically obtain the influence of surface energy on particle deposition, the relationship between the particle velocity, particle height and surface energy was investigated in the study. As shown in Fig.5, it can be found that particle obtain higher rebound velocity and rebound height after colliding with surface with lower surface energy than that with bigger surface energy. This is because that the adhesion force between particle and super-hydrophobic surface is lower due to the lower surface energy. The particle may be easier to rebound off the bottom of the horizontal pipe and leave the pipe.

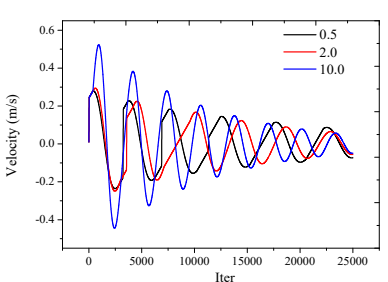

(a) Particle velocity

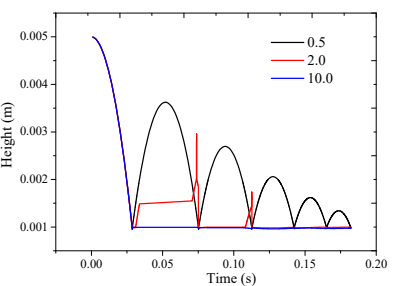

(b) Particle height
Fig. 4. The velocity and height of the particles different surface energy during the deposition process.

\subsubsection{Effect of the particle diameter on particle deposition}

Particle diameter plays an important role in particle deposition process. Different particles show different deposition characteristics on the surface in real environment. Thus the influence of particle diameter on dust deposition was analyzed in the study. It can be found that the small particles are easier to deposit on the super-hydrophobic surface while the larger particles are easier to rebound. The bounce times of small particles in the pipe were obviously less than large particles. To vividly reveal the collision process, the velocity and height of the particles with different diameters was displayed. The results in Fig.6 show that the rebound velocity of big particles is bigger than small particles and the rebound height is similar. This may be because that the bigger particles have higher kinetic energy when they collide with the surface. The influence of surface energy on small particle deposition process is more obvious than that on bigger particles thus the small particle would eventually adhere on the surface and can not leave the horizontal pipe.

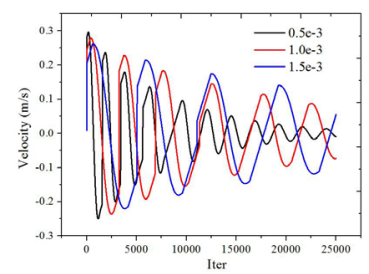

(a) Particle velocity

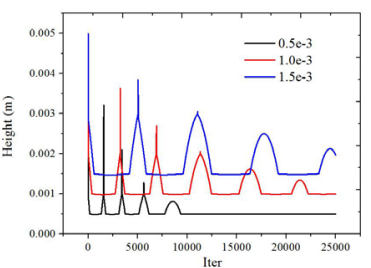

(b) Particle height
Fig. 5. The velocity and height of the particles different particle diameters during the deposition process.

\subsubsection{Effect of the Young's module on particle deposition}

The Young's module of the particle is the main mechanism factor of the particle deposition behavior. The particle would obtain a bigger rebound force with bigger Young's module during the process of deposition. The results in Fig. 6 show that the velocity and rebound height of the particle with larger Young's module are much larger than that the particles with small Young's 
module. Thus the particles with larger Young's module would not adhere on the surface during the deposition behavior and it is much easier for them to leave the horizontal pipe.

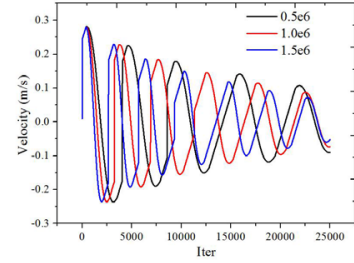

(a) Particle velocity

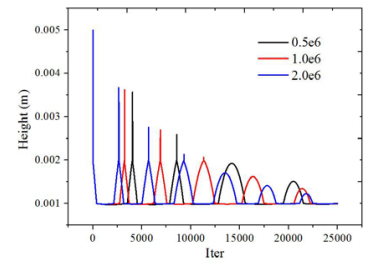

(b) Particle height
Fig. 6. The velocity and height of the particles Young's module during the deposition process.

\section{Conclusions}

The results show that the velocity and height of the particle are obviously affected by the surface energy of the surface, Young's module and diameters of the particle. It is easier for particles with greater Young's module, larger diameters and lower surface energy to rebound off the surface. In the future work, multiparticles in the system may be considered for numerical simulation to be related to the reality. Besides, the relationship between dust deposition and superhydrophobic surface can be investigated based on the parameters such as deposition density. The results obtained by dust deposition research can be helpful for the self-cleaning field.

\section{References}

[1] Z. A. Darwish, H. A. Kazem, K. Sopian, et al. Effect of dust pollutant type on photovoltaic performance [J]. Renewable and Sustainable Energy Reviews, 41, 735744 (2015)

[2] T. Sueto, Y. Ota, K. Nishioka. Suppression of dust adhesion on a concentrator photovoltaic module using an anti-soiling photocatalytic coating [J]. Solar Energy, 97, 414-417 (2013)

[3] D. F. Fan, B. H. Dong, S. M. Wang, et al. Research process of Self-cleaning technologies on solar panels [J]. Mater. Rev., 29, 111-115 (2015)

[4] V. Sharma, S. S. Chandel. Performance and degradation analysis for long term reliability of solar photovoltaic systems: a review [J]. Renew Sustain Energy Rev., 27, 753-767 (2013)

[5] A. J. Pan, H. Lu, L. Z. Zhang.. Experimental investigation of dust deposition reduction on solar cell covering glass by different self-cleaning coatings [J]. Energy, 185, 645-653 (2019).

[6] Z. G. Feng, E. E. Michaelides. The Immersed Boundary-Lattice Boltzmann Method for Solving FluidParticles Interaction Problems [J]. Journal of Computational Physics. 195, 602-628 (2014)
[7] S. Y. Chen, G. D. Doolen. Lattice Boltzmann method for fluid flows [J]. Annual review of fluid mechanics, 30, 329-364 (1998) 